\title{
Bladder Neuroendocrine Carcinoma: About Five Cases and Review of the Literature
}

\author{
Maryam Zaouit ${ }^{1}$, Farida Marnissi ${ }^{2}$, Hassan Jouhadi ${ }^{1}$, Nadia Benchekroun ${ }^{1}$, Asma Elkbir ${ }^{2}$, \\ Souha Sahraoui ${ }^{1}$ and Abdellatif Benider ${ }^{1}$
}

${ }^{1}$ Mohamed VI Center for Cancer Treatment, CHU Ibn Rochd Casablanca, Morocco

${ }^{2}$ Anatomopathology Service, CHU Ibn Rochd Casablanca, Morocco

*Corresponding author: Maryam Zaouit, Mohamed VI Center for Cancer Treatment, CHU Ibn Rochd Casablanca, Morocco

\begin{abstract}
Introduction: Primary neuroendocrine carcinoma of the bladder is an aggressive disease with significant metastatic potential. It is an extremely rare variation of non-urothelial carcinoma of the bladder, accounting for $<1 \%$ [1]. Given its rarity, no therapeutic consensus has been codified to date. We report a retrospective series of five cases and detail their anatomoclinical and therapeutic aspects.

Patients and methods: This is a retrospective descriptive study of five cases of neuroendocrine carcinoma of the bladder collected at the Mohammed VI Center, between January 2017 and December 2020. The diagnosis was confirmed by immunohistochemical study. The median age is 61 years (extremes: $36-73$ years), all patients are male. The average delay of consultation is 7 months (extremes: 2-12 months). Chronic smoking was found in three patients. Hematuria was the cardinal sign in 4 cases. Irritative urinary disorders such as pollakiuria and dysuria were noted in two cases. Cystoscopy showed a solid tumor in the majority of cases, located in the bladder trigone in two patients, and in the posterobasal wall in two others. Neuroendocrine carcinoma was pure in four cases and impure; associated with a poorly differentiated carcinoma component in only one case. The diagnosis of small cell neuroendocrine carcinoma of the bladder is retained after an immunohistochemical study. Chromogranin A was expressed in all cases and Synaptophysin in four cases. The extension work-up revealed locally advanced and immediately metastatic cancer in three patients (peritoneum, lung, skin). The performance status index was between 0 and 1. Palliative chemotherapy was recommended in three patients, two of whom refused all treatment. The other two patients received radiotherapy as adjuvant to neoadjuvant chemotherapy.
\end{abstract}

Conclusion: The incidence of neuroendocrine carcinomas of the bladder is increasing due to the diagnostic progress by immunohistochemical means available today. They are often diagnosed at a stage that is already locally advanced or metastatic; their evolution is very rapid and their prognosis is poor. At the present time, there is no therapeutic consensus for their management.

\section{Keywords}

Bladder, Neurocarinoma, Synaptophysin, Chromogranin A

\section{Introduction}

Primary neuroendocrine carcinoma of the bladder is an aggressive disease with significant metastatic potential. It is an extremely rare variation of nonurothelial bladder carcinoma, accounting for $<1 \%$ [1].

According to the WHO classification 2016, neuroendocrine carcinoma of the bladder includes; small cell neuroendocrine carcinoma (SCNEC) which is the most common subtype, large cell neuroendocrine carcinoma (LCNEC), paraganglioma and well differentiated neuroendocrine tumor [2-4].

Given its rarity, no therapeutic consensus has yet been codified.

We report a retrospective series of five cases of neuroendocrine carcinoma and we detail the anatomoclinical and therapeutic aspects through a study of the literature.

\section{Patients and Methods}

This is a descriptive retrospective study of five cases of neuroendocrine carcinoma of the bladder collected

Citation: Zaouit M, Marnissi F, Jouhadi H, Benchekroun N, Elkbir A, et al. (2022) Bladder Neuroendocrine Carcinoma: About Five Cases and Review of the Literature. Int J Oncol Res 5:036. doi.org/10.23937/2643-4563/1710036

Accepted: February 05, 2022: Published: February 07, 2022

Copyright: (C) 2022 Zaouit M, et al. This is an open-access article distributed under the terms of the Creative Commons Attribution License, which permits unrestricted use, distribution, and reproduction in any medium, provided the original author and source are credited. 


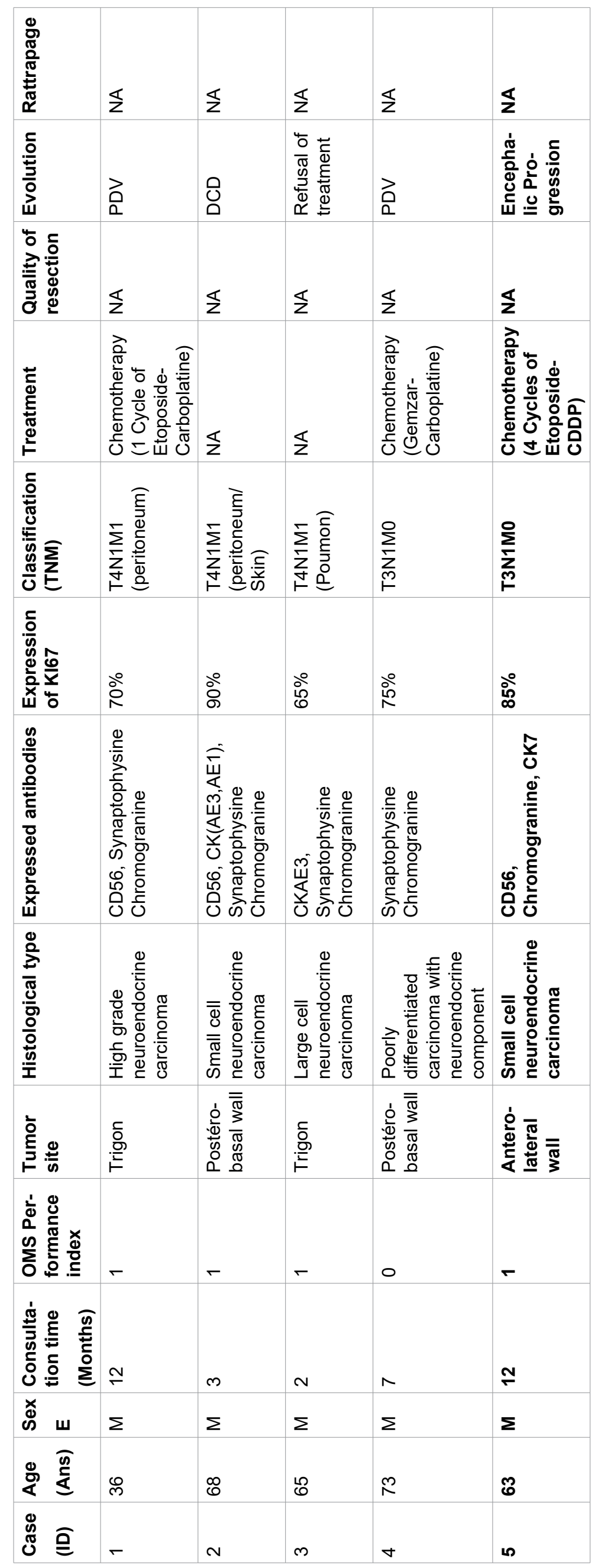


at the Mohammed VI Center for the Treatment of Cancers in Casablanca between January 2017 and December 2020. The diagnosis is confirmed by the immunohistochemical study including Les markers used are antichromogranin antibody, anti synaptophysin antibody, and anti-CD56. The stage of the disease is defined according to the Tumor Node Metastasis (TNM) classification of the American Joint Committee on Cancer (AJCC) in 2010. The chemotherapy used was based on a doublet comprising platinum salts.

The median age is 61 years (range: $36-73$ years), all patients are male. The average consultation time is 7 months (range: 2-12 months). The notion of chronic smoking is found in three patients. Hematuria is the cardinal sign in 4 cases. Irritative urinary disorders such as pollakiuria and dysuria are noted in two cases. Cystoscopy shows a tumor of solid appearance in the majority of cases, localized at the trigone of the bladder in two patients, and at the level of the postero-basal wall in 2 others. Neuroendocrine carcinoma is pure in four cases and impure, associated with a component of poorly differentiated carcinoma, in only one case.

The diagnosis of neuroendocrine small cell carcinoma of the bladder is made after an immunohistochemical study. Chromogranin A is expressed in all of our patients and Synaptophysin in four patients (Table 1).

The extension assessment (Figure 1) revealed locally advanced cancer that immediately metastasized in three patients (peritoneum, lung, skin). The performance index of the World Health Organization (WHO) is between 0 and 1 in our patients. Palliative chemotherapy is indicated in three patients (ID 1, 2 and $3)$, two of whom refuse all treatment (ID 1 and 3).

The other two patients (ID 4 and 5) received neoadjuvant chemotherapy followed by radiochemotherapy. Patient ID 4 received three courses of neoadjuvant chemotherapy of the Gemzar-Carboplatin type and was then lost to follow-up without being irradiated for his bladder cancer. Patient ID 5 progressed in the brain after four courses of chemotherapy of the Etoposide-Cisplatin type (Figure 2 and Figure 3).

Our patient ID5 received encephalic radiotherapy in toto using a three-dimensional conformational technique. Then he was referred to palliative care given the deterioration of his performance index of the World Health Organization. Patient and treatment data are presented in the Table 1.

\section{Discussion}

First described in 1981, neuroendocrine carcinoma of the bladder is a rare tumor representing less than $1 \%$ of bladder tumors $[1,2]$. The pathogenesis of these tumors is not clearly established. Three hypotheses were formulated. The most commonly accepted hypothesis would be their development from multipotent cells, present in the urothelium, which are capable of differentiating into glandular, squamous or endocrine cells. However; other authors such as Cramer, et al. suggested the hypothesis of a metaplasia of the transitional epithelium [5]. Pure forms have been reported in over $50 \%$ of cases and are often more common than mixed forms [6].

This malignancy generally affects male patients aged 50 to 80 years $[7,8]$, although it can be observed in patients aged 35 to 87 years according to the current literature [9].

Neuroendocrine carcinoma of the bladder may be associated with long-lasting cystitis, gallstones and smoking $[10,11]$, previous studies have shown that smoking is the most important risk factor $[12,13]$. The clinical presentation is not very specific; hematuria is the most common telltale sign [14], which can be

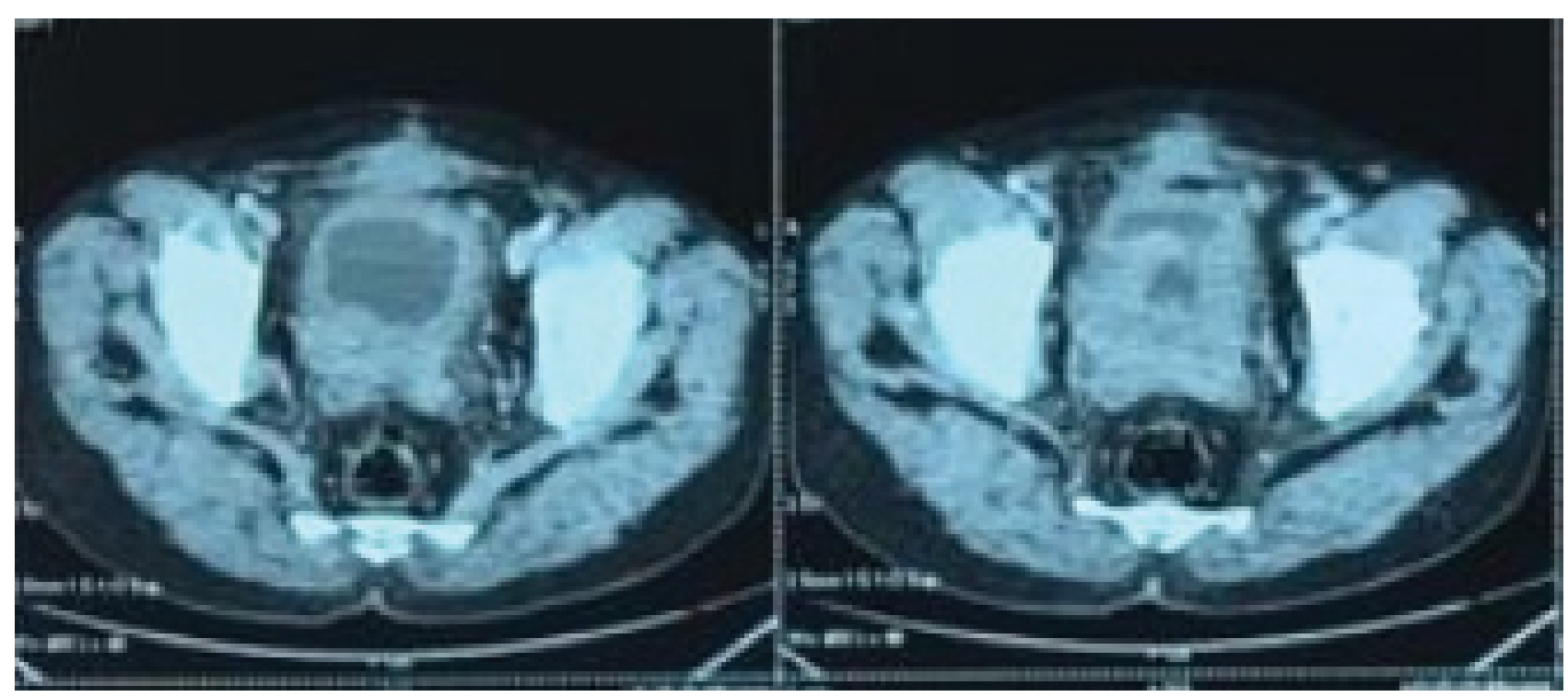

Figure 1: Sagittal section of a pelvic CT scan showing the bladder process (ID5). 

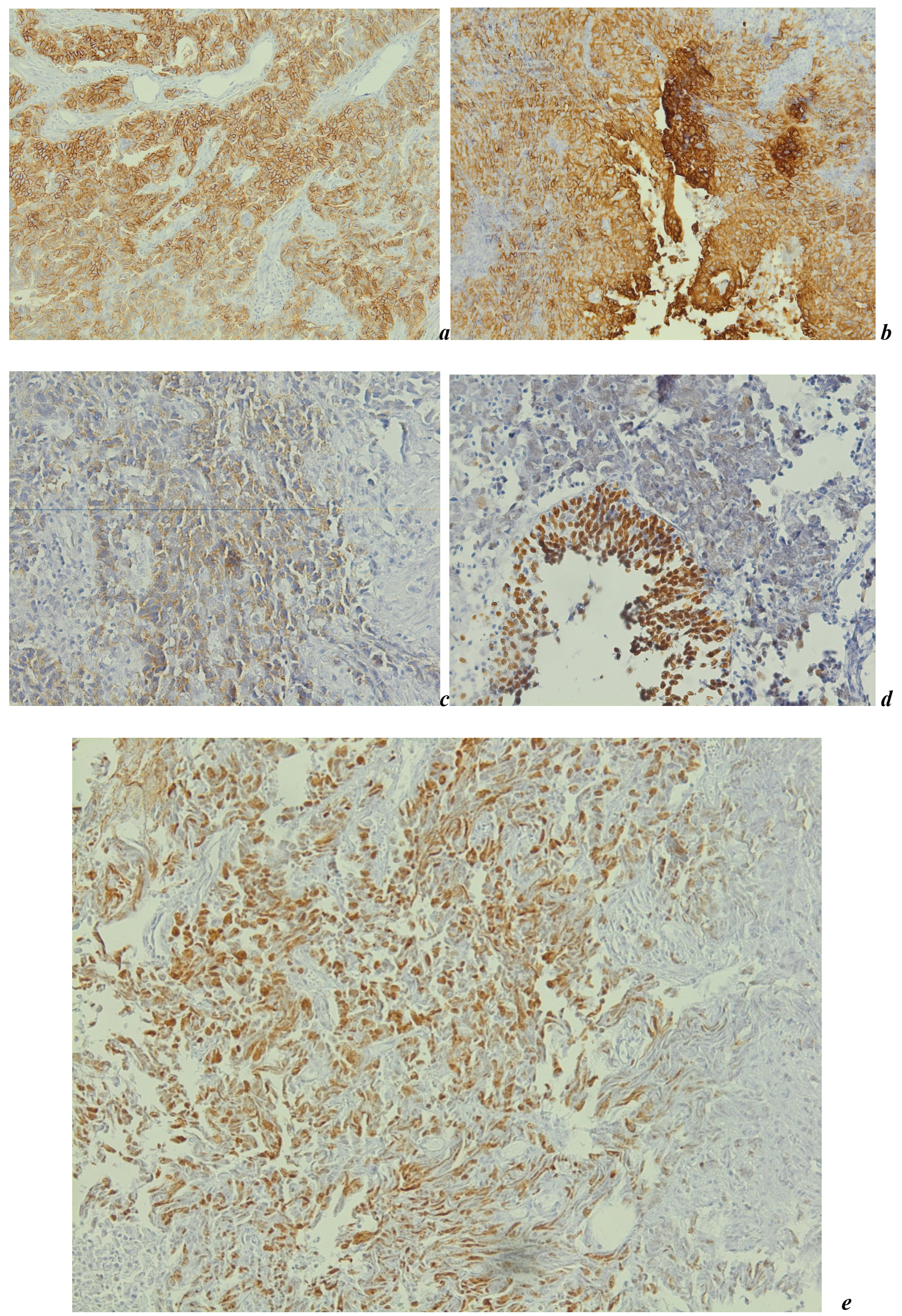

Figure 2: The tumoral cells are positive for P63, CK7, Chromogranine, and $\operatorname{CD} 56$ (a,b,c and d). (e) The Ki67 is hight, estimated to $85 \%$. 


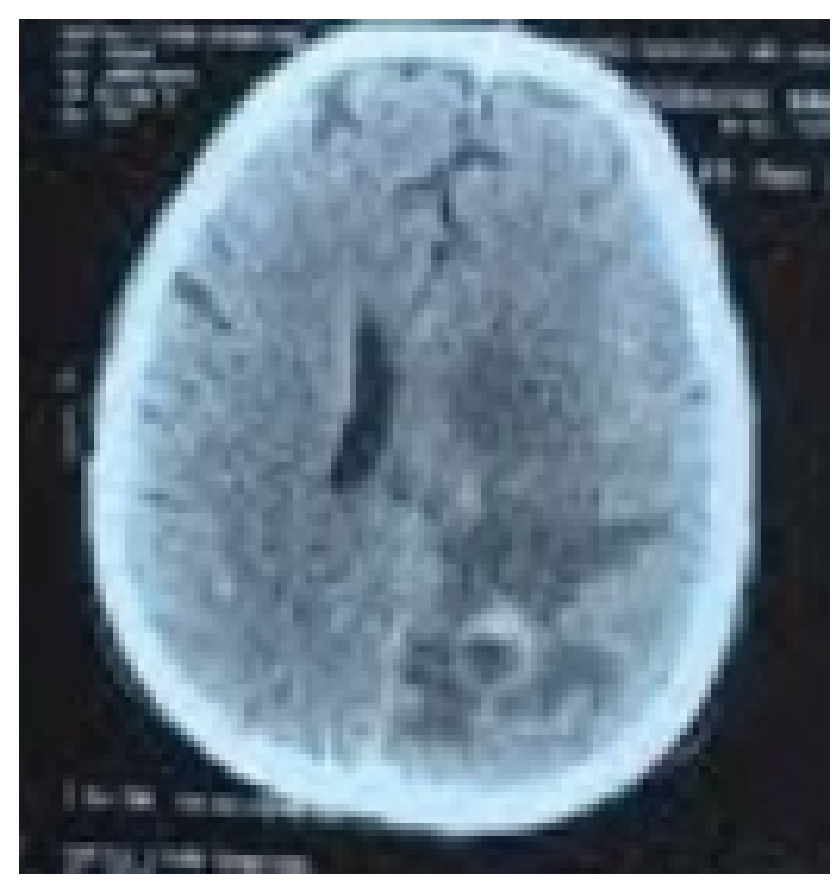

Figure 3: Sagittal section of a brain CT scan showing a left parietal lesion associated with perilesional edema (ID5).

accompanied by pain, and irritative urinary tract disorders. These epidemiological findings were found in our series.

The definitive diagnosis is based on the anatomopathological study supplemented by an immunohistochemical study. Chen, et al. verified that CD56, Synaptophysin, neuron-specific-enolase and $\mathrm{CgA}$ are positive and sensitive markers for neuroendocrine carcinoma of the bladder [15], which was consistent with the immunohistochemical results in our present series.

The stage of the disease is defined by the TNM classification of the American Joint Committee on Cancer (AJCC) of 2010. But some authors recommend distinguishing between localized tumors and disseminated tumors, and not to use the TNM classification, which seems not to be not suitable for this type of tumor [16]; as is the case with small cell carcinoma of the lung. In our series, the diagnosis was made when the tumor was locally advanced and metastatic in three patients.

Due to the limited amount of published data on neuroendocrine carcinoma of the bladder and the lack of randomized trials; therapeutic modalities are not yet well established. Multimodal treatment including surgery, chemotherapy and radiotherapy was most frequently recommended [17]. Recent studies have shown that patients with neuroendocrine carcinoma of the bladder who underwent cystectomy, chemotherapy and radiotherapy, had better OS and CSS $[3,18]$ compared to patients who received unimodal treatment. Therefore; the chemotherapy protocol had to include a doublet based on platinum salts to improve the survival rate; as reported by Bhatt, et al. [19].
In the light of these data from the literature which highlight the role of systemic treatment in this disease, neoadjuvant chemotherapy is recommended before any local treatment in the disease localized to the pelvis. Most of the authors recommended first-line surgery and radiotherapy was often reserved for inoperable patients and/or unresectable or salvage tumors after a local relapse $[6,20]$ since the rate of local relapses after radiotherapy has been reached. $60 \%$ at 3 years according to some studies [21].

Regarding prophylactic brain irradiation (PCI), some discuss it from the advanced stages (III and IV), where the disease is well controlled [22]. But randomized trials are needed to confirm the benefit of this irradiation.

However; despite therapeutic progress; the median survival time in these patients is less than 9 months, and the overall five-year survival rate for all stages is $19 \%$ $(16-25 \%)[23,24]$.

\section{Conclusion}

The incidence of neuroendocrine carcinomas of the bladder is increasing due to the diagnostic progress by immunohistochemical means available to date. Often diagnosed at an already locally evolved or metastatic stage; they evolve very quickly and have a poor prognosis. At present, no therapeutic consensus has been codified for treatment.

\section{References}

1. Thota S, Kistangari G, Daw H, Spiro T (2013) A clinical review of small-cell carcinoma of the urinary bladder. ClinGenitourin Cancer 11: 73-77.

2. Humphrey PA, Moch $\mathrm{H}$, Cubilla AL, Ulbright TM, Reuter VE (2016) The 2016. WHO classification of tumors of the urinary system and male genital organs-part B: prostate and bladder tumors. Eur Urol 70: 106-119.

3. Niu Q, Lu Y, Xu S, Shi Q, Guo B, et al. (2018) Clinicopathological characteristics and survival outcomes of bladder neuroendocrine carcinomas: a population-based study. Cancer Manag Res 10: 4479-4489.

4. Colarossi C, Pino P, Giuffrida D, Aiello E, Costanzo R, et al. (2013) Large cell neuroendocrine carcinoma (LCNEC) of the urinary bladder: a case report. Diagn Pathol 8: 19.

5. Cramer SF, Aikawa M, Cebelin M (1981) Neurosecretory granules in small cell invasivecarcinoma of the urinary bladder. Cancer 47: 724-730.

6. Pasquier D, Barney B, Sundar S, Poortmans P, Villa S, et al. (2015) Smallcell carcinoma of the urinary bladder: a retrospective, multicenter Rare CancerNetwork study of 107 patients. Int J Radiat Oncol Biol Phys 92: 904-910.

7. Holmang S, Borghede G, Johansson SL (1995) Primary small cell carcinoma of the bladder: A report of 25 cases. $J$ Urol 153: 1820-1822.

8. Lau SK, Zhang Y (2004) Pathologic quiz case. A 73 year old man with a bladder mass. Combined primary small cell neuroendocrine carcinoma and highgrade papillary urothelial carcinoma of the urinary bladder. Arch Pathol Lab Me 128: 1055-1056. 
9. Nabi G, Singh I, Ansari MS, Sharma MC, Dogra PN (2001) Primary small cell neuroendocrine carcinoma of urinary bladder: An uncommon entity to be recognized. Int Urol Nephrol 33: 637-640.

10. Ahsaini M, Riyach O, Tazi MF, El Fassi MJ, Farih MH, et al. (2013) Small cell neuroendocrine carcinoma of the urinary tract successfully managed with neoadjuvant chemotherapy. Case Rep Urol 2013: 598325.

11. Alijo Serrano F, SanchezMora N, Angel Arranz J, Hernandez C, Alvarez Fernandez E (2007) Large cell and small cell neuroendocrine bladder carcinoma: Immunohistochemical and outcome study in a single institution. Am J Clin Pathol 128: $733-739$.

12. Asamura $H$, Kameya $T$, Matsuno $Y$, Noguchi $M$, Tada $H$, et al. (2006) Neuroendocrine neoplasms of the lung: a prognostic spectrum. J Clin Oncol 24: 70-76.

13. Rossi G, Cavazza A, Marchioni A, Longo L, Migaldi M, et al. (2005) Role of chemotherapy and the receptor tyrosine kinases KIT, PDGFRalpha, PDGFRbeta, and Met in largecell neuroendocrine carcinoma of the lung. J Clin Oncol 23: 8774-8785.

14. Choong NWW, Quevedo JF, Kaur JS (2005) Small cell carcinoma of the urinary bladder. The Mayo Clinic experience. Cancer 103: 1172-1178.

15. Chen Z, Liu Q, Chen R, Liu Z, Li M, et al. (2017) Clinical analysis of small cell carcinoma of the bladder in Chinese: Nine case reports and literature reviews. World J Surg Oncol 15: 33.

16. Ismaili N, Heudel PE, Elkarak F, Kaikani W, Bajard A, et al. (2009) Out-come of recurrent and metastatic small cell carcinoma of the bladder. BMC Urol 9: 4.
17. Ghervan L, Zaharie A, Ene B, Elec FI (2017) Small-cell carcinoma of the urinary bladder: where do we stand? Clujul medical 90: 13-17.

18. Xia K, Zhong W, Chen J, Lai Y, Huang G, et al. (2020) Clinical Characteristics, Treatment Strategy, and Outcomes of Primary Large Cell Neuroendocrine Carcinoma of the Bladder: A Case Report and Systematic Review of the Literature. Front Oncol 10: 1291.

19. Bhatt VR, Loberiza FR Jr., Tandra P, Krishnamurthy J, Shrestha R, et al. (2014) Risk factors, therapy and survival outcomes of small cell and large cell neuroendocrine carcinoma of urinary bladder. Rare Tumors 6: 5043.

20. Lynch SP, Shen Y, Kamat A, Grossman HB, Shah JB, et al. (2013) Neoad-juvant chemotherapy in small cell urothelial cancer improves pathologicdownstaging and long-term outcomes: results from a retrospective study atthe MD Anderson Cancer Center. Eur Urol 64: 307-313.

21. Lohrisch C, Murray N, Pickles T, Sullivan L (1999) Small cell carcinoma of the bladder: long term outcome with integrated chemoradiation. Cancer 86: 2346-2352.

22. Ismaili N (2011) A rare bladder cancer - small cell carcinoma: review and update. Orphanet J Rare Dis 6: 75.

23. Elktaibi A, Nasri A, Oukabli M, Bernoussi Z, Bouzidi AA, et al. (2013) Small cell neuroendocrine carcinoma of the bladder: a rare and aggressive anatomoclinical entity. J Afr Cancer Afr J Cancer 5: 237-239.

24. Macedo LT, Ribeiro J, Curigliano G, Fumagalli L, Locatelli $M$, et al. (2011) Multidisciplinary approach in the treatment of patients with small cell bladder carcinoma. Eur J Surg Oncol 37: 558-562. 